\title{
Prevalence of Sleep Disorders among Children in Elementary Schools in Kirkuk City
}

\author{
Abbas Lateef Muhe-Aldeen * Radhwan Hussein Ibrahim **
}

\begin{abstract}
Background and aim: the purpose of this study was to examine the prevalence rates of several symptoms of sleep disorders in young children, and the relationship between symptoms of pediatric sleep disorders and other childhood problems.

Materials and method: a descriptive correlation study design , subjects consist of (600) pupils divided in to 310 male and 290 female, aged 6 to 12 years, The data were collected from 20 Kirkuk elementary schools during a period of five months extending from December, $4^{\text {th }}, 2011$ to May, $6^{\text {th }}, 2012$. The data had been collected through the use of the developmental questionnaire and the interview technique with the family of pupils in the school. Reliability and validity of questionnaire were determined by performance of a pilot study. Data were analyzed through the application of descriptive statistics (percentage, ANOVA and mean) and inferential statistic analysis (correlation coefficient and chi-Square test). Children who rated as high risk for having sleep disorder displayed more sleep behaviour and waking during the night.
\end{abstract}

Results: It was found that (25\%) of children were at high risk for having at least one type of sleep disorder.

Conclusions: High rates of sleep disorder symptoms were observed across all disorder categories, particularly in Excessive daytime sleepiness and Obstructive Sleep Apnea Syndrome. This indicates that the symptoms of sleep disorders are already apparent even at sample $(6-12)$ years old.

Keywords: Prevalence, Children, Sleep Disorder.

\section{INTRODUCTION}

Sleep is as important to the human body as food and water, Lack of sleep can also affect a child's school performance and could be linked to increased risk of emotional problems such as depression. Adequate sleep is important for optimum human health, and is necessary for memory stabilization and consolidation (Diekelmann and Born, 2010; Yoo et. al. 2007). Children aged $(5-12)$ need 10-11 hours of sleep. At the same time, there is an increasing demand on their time from school (e.g., homework), sports and other extracurricular and social activities. In addition, school-aged children become more interested in TV, computers, the media and Internet as well as caffeine products - all of which can lead to difficulty falling asleep, nightmares and disruptions to their sleep. In particular, watching TV close to bedtime has been associated with bedtime resistance, difficulty falling asleep, anxiety around sleep and sleeping fewer hours (Donaldson and Owens, 2006). Sleep problems and disorders are prevalent in children. Poor or inadequate sleep can lead to mood swings, behavioral problems such as hyperactivity and cognitive problems that impact on their ability to learn in school (Archbold et. al., 2002). In Saudi Arabia, $8 \%$ of the children have sleep disorders, and in Egypt, 9\% of the children have sleep disorders (Ohayon, 2002). In Iran, a study reported a prevalence of sleep problems in school-aged children in Tehran was : bedtime problems $(21.05 \%)$; excessive daytime sleepiness (42.98\%); awakening during the night $(32.46 \%)$; regularity and duration of sleep (17.54\%); sleep disordered breathing (10.53\%) (Mahmoud Mohammadi MD (Mahmoud et. al, 2007). In Libya, a study reported a prevalence of sleep disorder is $(28.9 \%)$ (AL-Sharbati, 2002). In Turkey, a study reported a prevalence of sleep disorders in preadolescent school-aged children is (14.4\%) (Mehmet, 2004).

The primary purpose of this study was To assess the prevalence of school children who display indicative symptoms of sleep disorders, and to determine whether there is a relationship between children who are at risk for sleep disorders as measured by the Sleep Disorders Inventory for Students (SDIS), Children's Sleep Habits Questionnaire ( CSHQ) .

\section{MATERIALS AND METHOD}

A descriptive correlation study was carried out from September, 19 ${ }^{\text {th }}, 2011$ to

\footnotetext{
* Assistant Lecturer / College of Nursing / University of Kirkuk.

** Professor / College of Nursing / University of Mosul.
} 
August, $1^{\text {st }}, 2012$ in order to achieve the objectives of the present study. The whole study population consisted of 104183 pupils who were distributed among 312 schools. A probability sample (random) consisted of 600 pupils were chosen from (20) schools, The sample age ranges between ( $6-12$ ) years. The study tools was developed by the researcher to achieve the purpose of this study. It consisted of Children's Sleep Habits Questionnaire (CSHQ), and sleep Disorders Inventory for Students (SDIS). The CSHQ contain 22 item represent parent answer to the questionnaire (Owens et. al., 2000). it has been used in a number of studies to examine sleep habits in young children. CSHQ consist of five parts (Demographic data sheet, Bedtime, Sleep behaviour ,Waking during the night, Morning waking up). Parents are asked to recall sleep behaviors occurring over a "typical" recent week. Unless noted, check (Always) if something occurs every night, (Usually) if it occurs 5 or 6 times a week, (Sometimes) if it occurs 2 to 4 times a week (Rarely) if it occurs once a week, and (Never) if nothing there is occurs. The Demographic data sheet was developed by the researcher in order to collect additional information about the participants in this study. The Demographic data sheet include questions about school names, child's gender, age , grade (class), address, and sector. The Bedtime consist of nine items that focus on child bedtime habits for example "Child goes to bed at the same time at night". The Sleep Behaviour it consist of seven items that focus on child sleep behaviour for example " Child grinds teeth during sleep". The Waking during the night it consist of two items that focus on child waking during the night for example " Child wakes up once during the night". The Morning wake up it consist of four items that focus on child morning wake up for example " Child wakes up by him/ herself ". So, the Sleep Disorders Inventory for Students SDIS (Luginbuehl, 2008), was developed in order to respond to the need for a school-based screening instrument to recognize the sleep disorders of Obstructive Sleep Apnea Syndrome (OSAS), Narcolepsy (NARC), Periodic Limb Movement Disorder (PLMD),
Restless Leg Syndrome (RLS), and Delayed Sleep Phase Syndrome (DSPS). The SDIS-C is available in both English and Spanish and is translated to Arabic. There are 37 behavioral questions answered by two option (Yes or No). Parents were asked to complete the SDIS-C to the best of their abilities, based on their child's sleep behavior during the past 6 to 12 months. If parents are unsure how to answer any of the questions, they are instructed to observe their child sleep on 2 different nights for 2 hours, a few hours after the onset of sleep and then again at 4:00 to 5:00 in the morning, preferably on a night during which the child is not taking any medication. This tool was chosen because of its specific design for children of this age and its technical properties. The responses of the SDIS-C were evaluated through a computerized scoring program which provides a range of (normal, cautionary, or high risk) on each of the sleep disorders for which this tool screens. A letter was sent through the school managers to the parents of children in order to explain the study. Parents who were able to observe their child's sleep and behavior for at least the past six and three months, respectively, were eligible for participation in this study. Parental consent forms and a demographic information sheet were given to the parents upon arrival to the screening meeting, and the researcher was present as well to answer any additional questions. In addition, the researcher explained to the parent specifically what the SDIS-C and CSHQ measure and how to complete this tool. The researcher was available to answer questions from the parents. After the parent finished completing these forms, the forms were returned to the researcher at the arrival desk and given to the researcher. The researcher completed the scoring of the SDIS-C and generated a computerized print-out of the results. The data had been collected through the use of the developmental questionnaire and the interview technique with the family of pupils in the school as a means of data collection process. Each parent spends approximately (10-15min ) to respond to the interview .

\section{RESULTS}

Table (1) Children Sleep Habits Questionnaire(CSHQ) Descriptive Statistics :

\begin{tabular}{|l|c|c|c|c|}
\hline Subscale & $\mathbf{X}$ & SD & Skewness & Kurtosis \\
\hline Bedtime & 91.9 & 17.82 & -1.07 & 1.22 \\
\hline Sleep behavior & 101.01 & 12.81 & -0.89 & 1.17 \\
\hline Waking during the night & 99.06 & 13.77 & -0.75 & -0.07 \\
\hline Morning wake up & 95.08 & 15.40 & -0.86 & 1.22 \\
\hline
\end{tabular}


The table shows that the means and standard deviations for (CSHQ) were as follows : (101.01 \pm 12.81) sleep behavior, (99.06 \pm 13.77$)$ waking during the night, $(95.08 \pm 15.40)$ morning wake up , and $(91.9 \pm 17.82)$ bedtime.

Table (2) Prevalence of Sleep Disorders as Measured by the SDIS-C.

\begin{tabular}{|l|c|c|c|c|}
\hline \multirow{2}{*}{ SDIS-C } & Normal & Caution & High risk & \multirow{2}{*}{ Total } \\
\cline { 2 - 4 } & $\mathbf{\%}$ & $\mathbf{\%}$ & $\mathbf{\%}$ & \\
\hline Overall & 75 & 12 & 13 & $100 \%$ \\
\hline OSAS & 81.40 & 6.98 & 11.62 & $100 \%$ \\
\hline PLMD & 83.70 & 8.15 & 8.15 & $100 \%$ \\
\hline DSPS & 82.58 & 6.93 & 10.49 & $100 \%$ \\
\hline EDS & 70.97 & 8.25 & 20.78 & $100 \%$ \\
\hline $\mathbf{X}^{2}=342.895$ & DF $=8$ & P.Value $=0.05$ \\
\hline
\end{tabular}

The table shows that (75\%) of children scored in the normal range of sleep. However, (12\%) of children received sleep scores in the cautionary range, and ( $13 \%)$ scored in the high-risk range. $(11.62 \%)$ of them had high risk for obstructive sleep apnea syndrome, $(8.15 \%)$ had periodic limb movement disorders, $(10.49 \%)$ had delayed sleep phase syndrome, and $(20.78 \%)$ had excessive daytime sleepiness.

Table (3): SDIS-C Subscale Percentages:

\begin{tabular}{|l|c|c|c|c|c|}
\hline \multirow{2}{*}{ Subscale } & \multicolumn{2}{|c|}{ Male } & \multicolumn{2}{c|}{ Female } & \multirow{2}{*}{ Total } \\
\cline { 2 - 5 } & No. & $\%$ & No. & \% & \\
\hline Normal & 244 & 40.7 & 196 & 32.7 & $73.4 \%$ \\
\hline Caution & 35 & 5.8 & 10 & 1.7 & $7.5 \%$ \\
\hline Risk & 45 & 7.5 & 70 & 11.6 & 19.1 \\
\hline Total & 324 & 54 & 276 & 46 & $100 \%$ \\
\hline $\mathbf{X}^{2}=19.321$ & DF $=2$ & P.Value $=0.05$ & \\
\hline
\end{tabular}

The table revealed that (73.4\%) of the children scored in the normal range across all sleep disorders areas. Further analysis revealed that $(7.5 \%)$ of the sample had a moderate risk of having at least one type of sleep disorder. The remainder of the children, (19.1\%), were at high risk for having at least one type of sleep disorder.

\section{DISCUSSION}

The results of this study indicated that $(25 \%)$ of children in this sample received a score of high risk in at least one category of sleep disorders. In this sample, particularly high rates of sleep disorders were found in the areas of Excessive Daytime Sleepiness/narcolepsy (29.03\%), Obstructive Sleep Apnea Syndrome $(18.6 \%)$, Delayed Sleep Phase Syndrome (17.42\%), and Periodic Limb Movement Disorder was not quite as prevalent as the other types of sleep disorders, but still occurred in a relatively high percentage of the sample (16.3\%) - While the majority of children had normal sleep overall (75\%) Table (4.3) show the Prevalence of Sleep Disorders as Measured by the SDIS-C . The Daytime fatigue was the most prevalent sleep problem $(37.5 \%)$ followed by bed time resistance (26.2\%), difficulty rising in the morning during weekdays (20.7\%), and sleep onset delay (11.8\%). Co-sleeping with parents was reported in $(12.4 \%)$ of children
(Ahmed et. al., 2006). The overall prevalence of sleep disorders was (21.2\%) and included parasomnia symptoms bruxism (6.5\%), sleep talking (4.9\%), enuresis (1.0\%), sleepwalking $(0.6 \%)$, restless sleep $(5.0 \%)$, and leg movements $(1.9 \%)$ and symptoms of sleepdisordered breathing frequent snoring (5.6\%), mouth breathing $(4.1 \%)$, choking / gasping $(0.9 \%)$, and breathing pauses $(0.2 \%)$ (Xicheng Liu et. al., 2005). The sleep starts, confusional arousal, sleep talking, bruxism, sleepwalking, sleep terrors, nightmares, sleep paralysis and nocturnal enuresis. Psychiatric diagnosis was determined from case record survey of the participating subjects, $62.2 \%$ of the children suffered from at least one problem, Girls were significantly more likely to suffer from sleep problems when compared with boys, There were significant gender differences associated with 2 sleep problems; while girls suffered significantly more from nightmares when compared with boys, boys suffered significantly more from 
nocturnal enuresis (Mahendran et.al., 2006). So, In this sample, particularly high rates of sleep disorders were found $(73.4 \%)$ of the children scored in the normal range across all sleep disorders areas, $(7.5 \%)$ of the sample had a moderate risk of having at least one type of sleep disorder and (19.1\%) of the children were at high risk for having at least one type of sleep disorder show in table (4.3) the SDIS-C Subscale Percentages. This study particularly supported the study about Sleep problems as follows: bedtime problems (21.05\% - 56.44\%), excessive daytime sleepiness $(26.73 \%-42.98 \%)$ , awakening during the night $13.86 \%$ $32.46 \%$, regularity and duration of sleep $17.54 \%$ - $27.72 \%$, sleep disordered breathing $10.53 \%$ $17.82 \%$ (Mahmoud et. al., 2007). Also, the overall prevalence of snoring was found to be $12.7 \%$ (Males $=12.8 \%$, Females $=12.6 \%$ ). The overall prevalence of sleep disordered breathing (SDB) was $4.8 \%$. Approximately $24.3 \%$ of the children (26.3\% males and $22.9 \%$ females) were seen to suffer from excessive daytime sleepiness. Sleep deprivation $(32.2 \%)$ was found to correlate positively with age $(\mathrm{p}<0.001)$. The overall prevalence of bedtime resistance was found to be $25.8 \%$. About $41 \%$ (Males= $41.1 \%$, Female $=40.9 \%$ ) of the children experienced nightmare once in a while. Amongst all subjects, $5.8 \%$ suffered from night terrors. About $12 \%$ of the entire population $(13.3 \%$ males and $10.4 \%$ females) were seen to suffer from bruxism. Approximately $7.7 \%$ of the overall population was observed to suffer from bedwetting. Insomnia was found in $17.3 \%$ of the subjects. On the whole, approximately $47.5 \%$ of the children were observed to suffer from some form of sleep disorder (Raphaële et. al., 2010). Sleep problems in Dutch children were present in $25 \%$, e.g. comparable to other populations, Sleep habits were age related, Problem sleepers scored significantly higher on all scales (Waleed et. al., 2011). The majority (58.3\%) of students went to bed before midnight and $18 \%$ of the total sample woke up before $6 \mathrm{am}$. Sleep latency of more than one hour was present in $19.3 \%$ of the students. Two thirds (64.8\%) of the students reported having at least one nocturnal awakening per night. Nightmares were the most common parasomnia reported by students. Daytime naps were common and reported in $74.5 \%$ of the study sample. Sleep quality was reported as "poor" in only $9.8 \%$ and was significantly associated with sleep latency, frequency of nocturnal awakenings, time of going to bed, nightmares but not with academic achievement (Daniel et. al., 2011). Overall, the prevalence rates of sleep disorders in this sample were higher in OSAS than the rates expected based on previous research. The prevalence of the following sleep symptoms waslisted as follows: habitual snoring (10.9\%), witnessed sleep apnea (1.5\%), nocturnal enuresis $(5.1 \%)$, and sleep teeth grinding (20.5\%). The results of this study suggest that at-risk populations of children commonly suffer from a sleep disorder in addition to having other problems. Therefore, consistent and even universal screening of sleep disorders in children experiencing a wide range of difficulties should be considered best practice in order to improve the problem identification stage of the provision of psychological services (Dally, 2006). School psychologists are also called upon to consult with teachers and parents dealing with children who have disruptive or otherwise concerning behaviors. Appropriate behavior is often considered to be a prerequisite to learning in young children, and previous research has made the link between behavior and educational outcomes clear at all ages (Dally, 2006).

\section{CONCLUSIONS}

The results of this study show that a very high percentage of the sample are at high risk for having at least one type of sleep disorder. The study also concluded that high rates of sleep disorders symptoms were observed across all disorder categories, particularly in Excessive Daytime Syndrome, and Obstructive Sleep Apnea Syndrome. This indicates that the symptoms of sleep disorders are already apparent even at this young age of (6 - 12 ) years old. In addition, particularly high rates of sleep disorders were found in the Excessive Daytime Sleepiness/narcolepsy.

\section{RECOMMENDATIONS}

The study recommends that children with sleep disorders must receive interventions through sleep specialists, school psychologists can intervene through appropriate identification of the problem in the problem-solving process. Educating students and educators about the prevalence and negative effects associated with sleep disorders, and even advocating for altering the school day so that children are educated during the times in which they are most alert. Additional research is also needed to more fully explore the impact of sleep disorders, which are corrected through medical intervention. Furthermore, educational programs about the prevalence and the effect of sleep disorders to 
the children in elementary school such as informational programs, Television, training parent in educational setting, conference, etc. to facilitate the early identification and intervention prevention. Pediatricians, school personnel, and parents all refer children to sleep clinics; it is important that all of these groups receive training and are knowledgeable about sleep disorders. Finally, future research should focus on using larger samples in order to gain a more accurate picture of prevalence rates in this population.

\section{REFERENCES}

Ahmed BaHammam, Eiad Al Faris, Shaffi Shaikh, and Abdulaziz Bin Saeed.(2006). Prevalence of sleep problems and habits in a sample of Saudi primary school children. An Saudi Med. 26 (1): 7- 13.

AL-Sharbati MM. (2002). Sleep problems among pupils in Benghazi , Libya. Saudi Med J. 23.1105-1109.

Archbold, K., Pituch, K., Panahi, P., and Chervin, R. (2002). Symptoms of sleep disturbances among children at two general pediatric clinics. Journal of Pediatrics. 140(1). P.p. 97-102.

Dally, K. (2006). The influence of phonological processing and inattentive behavior on reading acquisition. Journal of Educational Psychology. 98 (2), 420-437.

Daniel K. Ng, Ka-li Kwok, Josephine M. Cheung, Shuk-yu Leung, Pok-yu Chow, Wilfred H. Wong, Chung-hong Chan and Jackson C. Ho. (2011). Prevalence of Sleep Problems in Hong Kong Primary School Children: A Community-Based Telephone Survey. CHEST. 128:1315 1323.

Diekelmann, S., and Born, J. (2010). The memory function of sleep. Nature Reviews Neuroscience. 11. 114-126.

Donaldson, D. L., and Owens, J. A. Sleep and sleep problems. In G. G. Bear and K. M. Minke (Eds.). (2006). Children's needs III: Development, prevention, and intervention. P.p. $25-1039$.

Luginbuehl, M. L. (2008). The pediatric sleep disorders: validation of the Sleep Disorders Inventory for Students. school psychology review. 37(3). P.p.409-431.

Mahendran R, Subramaniam M, Cai YM, and Chan YH. (2006). Survey of sleep problems amongst Singapore children in a psychiatric setting. Soc Psychiatry Psychiatr Epidemiol . 41. P.p. 669-73.

Mahmoud Mohammadi MD; Babak Ghalebaghi, MD; Mir Farhad Ghaleh Bandi,MD; Ebrahim Amintehrani, MD; Shahnaz Khodaie, MD; Shervin Shoaee, MD; Mahmoud Reza Ashrafi, MD. (2007). Sleep Patterns and Sleep Problems among Preschool and School-aged Group Children in a Primary Care Setting. Iran journal of Pediatric. 17. (3). P.p. 213-221.

Mehmet Agargun, Y.; Cilli, Ali Savas; Sener, Sahnur; Bilici, Mustafa; Ozer, Omer Akil; Selvi, Yavuz; Karacan, Elvan. (2004). The Prevalence of Parasomnias in Preadolescent School-aged Children: A Turkish Sample. Sleep: Journal of Sleep and Sleep Disorders Research. 27(4). 701-705.

Ohayon, M. (2002). Sleep and the elderly. $J$ Psychosom Res. 56 (5) : 32. P.p. 463-464.

Owens JA, Spirito A, McGuinn M. (2000).The Children's Sleep Habits Questionnaire (CSHQ): psychometric properties of a survey instrument for school-aged children. Sleep. 23. P.p.1043-51.

Raphaële Reine Lydie van Litsenburg and Ruth C. Waumans and Gerrit van den Berg and Reinoud J. B. J. Gemke. (2010). Sleep habits and sleep disturbances in Dutch children: a population-based study. Eur journal of Pediatr. DOI 10.1007/s00431010-1169-8 .

Waleed M Sweileh, Iyad A Ali, Ansam F Sawalha, Adham S Abu-Taha, Sa'ed H Zyoud and Samah W Al-Jabi. (2011). Sleep habits and sleep problems among Palestinian students. Child and Adolescent Psychiatry and Mental Health. 5:25.

Xicheng Liu, MD; Yuyan Ma, MMed Sci; Yizhuo Wang, MMed Sci; Qinbo Jiang, BS; Xiaochun Rao, MMed Sci; Xiuying Lu, BS; and Honghong Teng, BS. (2005). An Epidemiologic Survey of the Prevalence of Sleep Disorders Among Children 2-12 Years Old in Beijing, China journal of the American Academy of Pediatrics.115.P.p. 266.

Yoo, S.-S., Hu, P. T., Gujar, N., Jolesz, F. A., and Walker, M. P. (2007). A deficit in the ability to form new human memories without sleep. Nature Neuroscience. 10. P.p. 385-392. 